\title{
Partnered innovation to implement timely and personalized care: A case study
}

www.cambridge.org/cts

Cite this article: Proctor EK, McKay VR, Toker E, Maddox TM, Hooley C, Lengnick-Hall R, MacGibbon S, and Evanoff B. Partnered innovation to implement timely and personalized care: A case study. Journal of Clinical and Translational Science 5: e121, 1-7. doi: $10.1017 /$ cts. 2021.778

Received: 23 November 2020

Revised: 1 March 2021

Accepted: 22 March 2021

\section{Keywords:}

Translational research; innovation; partnership; implementation; personalized care

\section{Address for correspondence:}

E. K. Proctor, PhD, \#1 Brookings Drive, St. Louis, Missouri, 63130. Email: ekp@wustl.edu

\section{Implementation, Policy and Community Engagement Research Article}

\section{Enola K. Proctor ${ }^{1}$ (1), Virginia R. McKay ${ }^{1}$, Emre Toker $^{2}$, Thomas M. Maddox ${ }^{3}$, Cole Hooley ${ }^{4}$, Rebecca Lengnick-Hall ${ }^{1}$, Simon MacGibbon ${ }^{5}$ and Bradley Evanoff ${ }^{6}$}

${ }^{1}$ Brown School, Washington University in St. Louis, Saint Louis, MO, USA; ${ }^{2}$ Washington University Medical School in St. Louis, Saint Louis, MO, USA; ${ }^{3}$ Healthcare Innovation Lab, BJC HealthCare/Washington University School of Medicine, Saint Louis, MO, USA; ${ }^{4}$ School of Social Work, Brigham Young University, Provo, UT, USA; ${ }^{5}$ Myia Labs Inc., San Francisco, CA, USA and ${ }^{6}$ Division of General Medical Sciences, School of Medicine, Washington University in St. Louis, Saint Louis, MO, USA

\begin{abstract}
Background: Understanding how to translate research discoveries into solutions for healthcare improvement is a priority of NIH-funded Clinical and Translational Science Awards (CTSA). This study, supported by one CTSA, aims to capture one process of shaping and implementing innovations to advance the timeliness and patient-centeredness of cardiovascular care. Specifically, we sought to understand a partnership between a private digital health startup company, a university innovation lab, and an academic health system's cardiology program pursuing this goal. Findings: The collaboration proceeded through clear phases to address the questions and challenges: problem definition, exploration and formalization of the partnership, innovation co-creation and pilot test, and scale-up planning. Phases were punctuated by key decisions, such as forming the partnership, negotiating terms of the partnership, iterating form and features of the innovation, and exploring sufficiency of its value-add for scale-up and sustainment. Key implementation concepts were apparent, including implementation strategies (e.g., champions and iterative trialing) and the implementation outcomes of acceptability, sustainment, and scale-up. Participants identified potential risks of collaboration, reflected on their co-creation process, and the value of engaging stakeholders in innovation design. Findings may inform subsequent collaborations between innovators and translational researchers. Methods: We conducted a case study to understand the partnership; characterize the questions they pursued, their decision points, information and data sources; and identify the challenges and risks. Data were collected through a series of four focus groups with members of each partnering organization. A transdisciplinary research team iteratively worked to condense and synthesize data from audio recorded transcripts into a case narrative.
\end{abstract}

(c) The Author(s), 2021. Published by Cambridge University Press on behalf of The Association for Clinical and Translational Science. This is an Open Access article, distributed under the terms of the Creative Commons AttributionNonCommercial-NoDerivatives licence (http:// creativecommons.org/licenses/by-nc-nd/4.0/), which permits non-commercial re-use, distribution, and reproduction in any medium, provided the original work is unaltered and is properly cited. The written permission of Cambridge University Press must be obtained for commercial re-use or in order to create a derivative work.

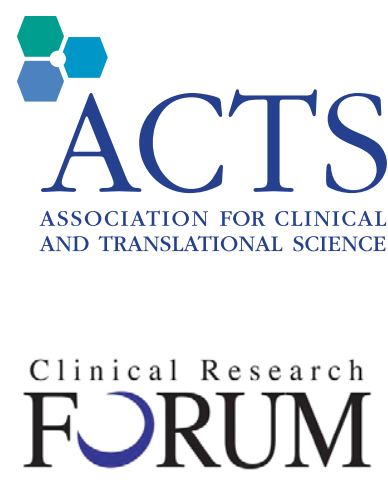

Analysis. Advocacy. Action.

\section{Introduction}

Unlike therapeutics and medical devices, innovations in healthcare delivery have been slow. Universities and health systems employ varied approaches to translate research discoveries into clinical and public health benefit. These include implementation science, quality improvement, technology transfer, and innovation labs. A growing number of health systems have established innovation labs, supported internally and charged with accelerating solutions to high priority problems. Innovation centers work to develop, test, and/or spread new diagnostics, therapeutics, and care delivery models in hospitals, clinics, and patients' homes [1]. Fundamentally, innovation is putting something new into practice, typically with intent to increase healthcare value [2,3]. Innovation centers often focus leveraging digital data and platforms [3]. Nearly three-fourths of large US healthcare systems have innovation centers, led by senior innovation officers [4].

The recent explosion of implementation science and its inclusion in CTSAs has intensified recognition of its role in translating medical discoveries into clinical care and community health improvements [5,6]. Implementation science emphasizes theory, systematic and reproducible methods including user-centered designs, and partnered approaches to developing and testing solutions. Implementation strategies include provider training, use of champions, and feedback via data dashboards [7,8]. Successful implementation is gauged by attainment of proximal outcomes such as feasibility, acceptability, adoption, and sustainment, which then are hypothesized to lead to improvements in clinical, service system, and population health benefits. Most hospitals also rely on quality improvement approaches such as Plan, Do, Study, Act (PDSA) cycles, occasionally blended with implementation science evaluation approaches [8].

Research translation also leverages technology transfer, commercial enterprise with industry partners, and spin-off companies. University technology transfer offices work to commercialize 
discoveries [9]. The primary objectives of entrepreneurship are the creation, delivery, and extraction of value typically from monetized products, services, or processes that others value more than alternative products - essentially the spin-off route. Market forces provide ultimate validation [9]. Entrepreneurs' strategies include trialing, conducting market viability assessments, prototyping, minimizing costs without degrading quality, and scaling successful innovations.

Innovation labs often partner with universities to apply implementation science principles to improve care delivery [10-13]. Innovation often requires partnering with third parties to realize gains. Navigating these partnerships is complicated, and little is known about best practices for these cutting-edge phenomena.

To address this void, we conducted a case study of one such partnership to:

- capture and describe the processes through which one innovation company and health system worked together to design and implement innovations into care delivery;

- characterize key questions, decision points, and challenges faced by these partners as they worked toward innovation uptake and scale-up;

- identify partnership and implementation science concepts that may guide other innovators, researchers and entrepreneurs.

The National Center for Clinical and Translational Science (NCATS) and the NIH-funded CTSAs have heightened visibility and support to translational research for system - and community - impact [6]. This study was supported by the Implementation Science-Entrepreneurship (IS-E) function of the CTSA at Washington University in St. Louis' (WUSTL), the Institute for Clinical and Translational Science (ICTS). The IS-E aims to advance understanding and synergy between these seemingly unrelated disciplines that share a focus on advancing the uptake and sustained delivery of new discoveries, albeit through different paths.

\section{Methods}

\section{Approach}

Case study methods enable in-depth exploration of new phenomena as they occur in natural real-life contexts $[14,15]$, thus yielding rich descriptions of this collaboration to co-create and pilot an innovation for cardiovascular care from private, academic, and clinical perspectives. Three organizations constituted the collaboration; key informants from each organization participated in the study. We maintain the confidentiality of individual participants, all of whom provided verbal consent. The Washington University Institutional Review Board approved study protocols.

\section{Organizations, Participants, and Roles}

The three organizations were a private digital health innovation company with a remote heart failure monitoring product (heretofore, XCo); the BJC HealthCare/Washington University School of Medicine (WUSM) Healthcare Innovation Lab; and the WUSM Cardiovascular Division.

The WUSM Cardiovascular Division is a national leader in high-quality cardiovascular care to a large, diverse patient population. With $75+$ faculty members, the Division conducts basic, translational, clinical, and population research to improve cardiovascular outcomes and has trained $300+$ cardiology fellows, graduate students, and post-doctoral research fellows.

The Cardiology Division was exploring passive and active remote monitoring of heart failure outpatients. Chronic heart failure is the most prevalent heart condition in the USA, requiring patient management of complicated medication regiments, frequent medical care, and significant change in lifestyle behaviors (health behavior, diet, environmental exposure, and physical activity). The Division wished to gather dynamic, longitudinal data from multiple sensors, in addition to physician- and patient-reported data. They focused on the usability, utility, and efficacy of the patient interface with sensors and mobile application, and of the clinician interface through the monitoring portal. WUSM cardiologists served as clinical informants and assessors of the technology.

The second organization is the BJC HealthCare/WUSM Healthcare Innovation Lab. The Innovation Lab was established as a cross-disciplinary organization to lead health system innovation. The Lab catalyzes care delivery innovations through research and development. In pursuit of this mission, the Lab leverages its academic association with WUSM to employ health services and implementation science research methods to validate rigorously its approach, support academic investigation and yield generalizable knowledge. The Lab employs clinicians, healthcare administrators, project managers, informaticians, and data analysts.

The Innovation Lab focuses on care delivery innovation, distinct from pharmaceutical or medical device innovation, given the need for improvements in the patient experience and outcomes in the US healthcare system and the emergence of novel care delivery models - many using digital tools - to meet that need. The Lab's programs engage dissemination and implementation researchers to study the development, implementation, and sustainability of its projects. It underwrites an internal grant program to support researchers with novel care delivery innovation ideas.

Two informants from the Innovation Lab participated. One was an administrator responsible for setting strategy goals, to ensure goal alignment with the larger health system priorities, and externally represent the organization. A second was responsible for coordinating activities and facilitating communication between the cardiology team at WUSM and digital health company.

The third organization was a private digital health company (henceforth referenced as XCo) whose mission is to optimize patient health technological solutions using data driven methods. XCo is an early stage start up with 30 employees. The company's virtual care software platform aims to help providers manage patients remotely from the home. XCo's solutions strive to modernize care for patients with chronic disease (e.g., Heart Failure, COPD, Diabetes, Hypertension) and other clinical conditions benefitting from extending care to the home. Platforms encompass remote patient monitoring, proprietary machine learning models, and a clinician application for managing a patient panel. The XCo system surfaces actionable physiological and symptom information and orchestrates timely patient interactions and care pathways - bringing clinicians and coaches into the loop when patients need them most. To move chronic care management toward prevention and personalized care, $\mathrm{XCo}$ emphasizes trust of patients and clinicians by using partnerships and co-design approaches.

Two company informants participated: one in an administrative role responsible for cultivating investment in XCo and building partnerships with potential adopting providers and a second responsible for managing deliverables and coordinating activities within XCo, providing regulatory expertise, and promoting the innovation. 


\begin{tabular}{|l|l|}
\hline KEY QUESTIONS AND CHALLENGES & $\begin{array}{l}\text { DECISIONS REACHED THROUGH } \\
\text { COLLABORATION }\end{array}$ \\
\hline $\begin{array}{l}\text { Are our goals compatible?Do we agree } \\
\text { how to better monitor chronic heart failure } \\
\text { patients? }\end{array}$ & $\begin{array}{l}\text { Cardiac monitoring requires innovative, } \\
\text { acceptable, and personalized solutions }\end{array}$ \\
\hline Should we form a partnership? & $\begin{array}{l}\text { Acknowledging advantages and risks, XCo and } \\
\text { the Innovation Lab decided to formally partner } \\
\text { and began working together in mid-2018. }\end{array}$ \\
\hline What are the terms of our partnership? & $\begin{array}{l}\text { The partnering organizations developed a formal } \\
\text { contract }\end{array}$ \\
\hline $\begin{array}{l}\text { What are the optimal features/functions of } \\
\text { the innovation? }\end{array}$ & $\begin{array}{l}\text { Partners determined that the innovation's form } \\
\text { and function would develop iteratively through a } \\
\text { series of pilots designed to capture user input }\end{array}$ \\
\hline Is the innovation worth scaling-up? & $\begin{array}{l}\text { Pilot outcomes would inform decisions about } \\
\text { value, sufficient for scale-up and spread. }\end{array}$ \\
\hline
\end{tabular}

Fig. 1. Challenges and decisions.

\section{Data Collection, Measures, and Analysis}

We conducted four focus groups with key informants, following a guide for each group. The topics were: (1) initiation of the collaboration and conception of the innovation; (2) process of innovation development; (3) risks and benefits of collaboration and a co-creation model; and (4) expectations for the future and ideal outcomes. Each focus group lasted approximately $90 \mathrm{~min}$, conducted at two- to four-week intervals to allow the research team to reflect on previous discussions to inform subsequent sessions. We collected data from November 2019-January 2020. At least one member from each organization was present for each focus group, with three to five participants per session. A member of the research team led each focus group; a note taker was present. Audio recordings of sessions were transcribed verbatim by a transcription company and reviewed for accuracy by a research team member.

Two research team members reviewed and coded transcripts and then discussed coding convergence until agreement was $100 \%$. The primary coder condensed and summarized data using a case narrative. All research team members reviewed and iteratively revised the final case summary.

\section{Results}

We present the results in narrative fashion to show the collaboration's development, highlighting challenges, questions requiring resolution, and the eventual decisions reached. Fig. 1 summarizes these questions and decisions, as detailed throughout the results, illustrated through exemplar quotes.

\section{Problem Definition, Goal, and Vision}

Determining compatibility among the partners' goals comprised the first challenge. XCo and the Innovation Lab prioritized the clinical issue of chronic heart failure, as did the Cardiology Division. This shared goal arose as a key topic by participants not from a focus group leader prompt. Participants discussed at length the nature of chronic heart failure and viewed clarity about the problem as a key first step to the viability of their partnership. They described this challenge and their perspectives as follows:

Cardiology Division participant: Close patient monitoring is beneficial, but it's been a challenge, to date, to find ways to monitor frequently enough to be able to understand if patients are starting to decline. Current strategies include asking patients to record daily weights and use phone calls. But because patients are physically removed from the clinical setting, and we need to follow them longitudinally ... More invasive strategies, like putting a sensor in someone has shown benefit, but is costly. Opportunities to intervene early and prevent decline are often missed. As a result, patients develop symptoms that are severe requiring emergency room visits and hospitalization. It is an extremely expensive condition to manage with our current protocols.

According to participants, the goal of the collaboration was to enable clinicians to accurately identify and intervene earlier for patients in decline using noninvasive monitoring devices and modern data transmission methods. Devices provide reliable and consistent monitoring at appropriate frequencies from patients at home or the workplace. An equally important goal was making the innovation appealing to those who will adopt it - clinicians and patients. As one XCo participant stated, “... if we can't get clinicians to adopt it, then it's all for nothing and there is no business."

Participants articulated a vision for timely, personalized healthcare in the future. Patients would have continuous experiences with early warning systems, would receive care prior to catastrophic events, and patient management would be optimized using timely information (e.g., medication titration). Patients also would be empowered for more proactive involvement in their care. Care would become more personalized via continuous, natural, and passive patient monitoring, requiring less direct supervision from clinicians. An XCo participant stated: "Everything will be de-averaged and personalized to the patient." Participants conveyed that developing the remote patient monitoring innovation would pave a pathway toward this vision.

Innovation Lab participant: You have a sort of shared vision for what the world will look like when you are done... in an industry like healthcare that ... requires transformation and change. So it's to have those people that have a vision for what the world will look like when we're done and have profound beliefs that are, unfounded ... and you're deploying something to test your hypothesis about those profound beliefs.

\section{Early Exploration of a Partnership}

Early on, XCo had launched an algorithm to identify heart failure patients using a social media platform and self-report data. Their initial attempt was mildly successful, providing strong proof of concept and evidence of feasibly collecting data from patients. Before marketing the algorithm, XCo wanted to demonstrate the 
- Running out of money before formal partnerships can be established, due to the slow pace of universities

- The partners might be poorly matched

- Reputations might be damaged

- Lack of familiarity with the market need might lead to efforts that are addressing the wrong problem

- Failure will impair future similar endeavors

Fig. 2. Partnership risks.

innovation's effectiveness and value and its feasibility and likelihood of adoption. The Innovation Lab, established to test interventions of potential to benefit patients, was already working with the WUSM Cardiology Division. XCo would be their first external partner. The parties agreed that conditions in the healthcare industry seemed right for capitalizing on emerging technology for remote sensors and monitoring.

Several aspects of this partnership appealed to XCo. The problem was clinically relevant, and it provided opportunity for passive data collection and co-creation via patient and clinician engagement. XCo had investments from reputable groups (e.g., the American College of Cardiology) but experienced challenges finding a reputable partner for formal co-design, pilot, and feasibility testing for a product so early in its development. At this stage of maturity, XCo found it essential to partner with health systems to seek "product market fit" with input from both patients and clinical users.

$\mathrm{XCo}$ : It provides legitimacy to an early stage business that otherwise no one knows anything about... we do not have a brand. We do not have a history of anything, other than our personal backgrounds... It brings a level of belief and trust [for] other customers and providers that are considering working with us.

Furthermore, this partnership would provide financial support. Funds reflecting the actual commercial pricing of the platform were essential to XCo's pursuit of venture capital to grow their business.

The Innovation Lab was already familiar with XCo's digital approach to solving clinical problems. The Innovation Lab was charged with trialing high risk/high reward innovations but lacked in-house technical expertise for innovation development. The Cardiology Division could provide access to a clinic and patient population to test and refine the innovation.

Along with these benefits, participants acknowledged the risks to partnering. The Innovation Lab believed that a more mature company might be more successful but would provide less flexibility in product design.

Innovation Lab: And ironically, I'm not totally sure I would have signed on to (XCo) today . . . .I'm not sure that's where we want to position the Lab. It may be, but there's been some discussions amongst our group about how mature do we want a company to be before we sign on as a partner. I think recently we've been gravitating a little bit towards more mature companies than where $[\mathrm{XCo}]$ is in its life cycle... there are some reasons to do that ... there are also some reasons not to do that. And one of them would be that you'd miss out on opportunities like [XCo].

Innovation Lab participants further noted the risk that a young company might fold after a short time. Fig. 2 lists other risks the participants identified.

\section{Formalizing a Relationship}

A formal partnership between the Innovation Lab and XCo carried legal and compliance challenges. The university typically worked with large, well-established companies, not early-stage companies. Most negotiations occurred between the between the Innovation Lab and XCo leaders, but the partnership had to be explained to their respective stakeholders (i.e., supervisors/board members) to help them see benefit and agree to the relationship. Legal issues of confidentiality, intellectual property, liability insurance, and human subjects' protections were negotiated formally. Partnership formalization took approximately 9 months, but leaders of this early stage business viewed entry to a large healthcare system and having a champion within the system as critical to their success and worthwhile. XCo valued the Innovation Lab's role as "an internal evangelist" to help boost the success of developing and piloting the innovation

\section{Co-Creating the Innovation}

Having formalized their partnership, XCo and the Innovation Lab proceeded to work collaboratively with the Cardiology Division to develop the innovation. The XCo placed high value on shaping a product that was not completely developed but reflected a vision of what it is intended to accomplish.

XCo: You've built a good portion of it, but the house is not finished... you've gotten the foundations down... you have a directional sense of what type of family you want the house to support. But you haven't chosen the furniture, you haven't chosen whether you're going to have blinds or drapes... these people live in a different way than you thought. So there is the need to bring something... but it ought not be finished.

Such a process would yield a product that is more likely to succeed. To XCo, the co-creation process itself was a primary benefit, particularly when there was no guarantee of this becoming a valuable commercial relationship. From the Lab's perspective, developing the innovation with XCo brought two advantages often absent in healthcare: speed in developing a solution and the ability to design with the end user in mind. Co-creation would ensure user input, leading to a better product that would be used with higher fidelity because users like it.

Focus group discussions revealed three steps in a cyclical process of co-creation: (1) understanding context, (2) synthesizing information, and (3) iterative prototyping. First, the partners worked to understand the clinical context for managing chronic heart failure by conducting observation in the field, watching clinicians doing what they do every day. As they described

First you've got to just observe and, and it uncovers a lot of opportunity.... Observation brings forth opportunities or parts of the problem you didn't realize were there in a way that is different from interviews or focus groups.

Observing the innovation in use in its natural setting - the cardiology clinic - was enlightening. For instance, they began with the idea of developing an early warning system, but talking to users helped them realize that clinicians were more interested in something that would help them provide personalized patient management. 


\begin{tabular}{|l|l|}
\hline Ideal outcomes: Innovation & Ideal outcomes: The partnership \\
\hline Proven effective through research & Will lead to innovation \\
\hline Feasible, desired by users, creates value & $\begin{array}{l}\text { Proves viability of partnering with } \\
\text { innovators }\end{array}$ \\
\hline Adds value & Elements of partnering are replicable \\
\hline Viable business model & Long term viability \\
\hline Clinicians will feel ownership in & Increases ownership for the business \\
\hline Elicits stakeholder buy-in & \\
\hline $\begin{array}{l}\text { Increases technology acceptance among } \\
\text { clinician }\end{array}$ & \\
\hline
\end{tabular}

Fig. 3. Ideal outcomes.

Lab: Some of the things that surfaced during the co-design effort were actually more about personalized patient management... for instance, in the observations we're seeing, you know, med changes happen and then, patient gets discharged. Oh, and then I [the physician] need to follow up with this ... patient in two weeks or next week. . . . Things get lost, you don't have timely data to make titration decisions....

XCo: It surfaces an opportunity, we hadn't thought of, . . . but now we can embed that into the solution and it changes our roadmap right? So I think that's one example of that really came to the surface as a result of this codesign quite early on, just through observation ...

Interviews with a broad set of stakeholders further yielded understanding of the organization context and goals, and important information about reimbursement mechanisms and other technology in the clinical setting.

XCo synthesized and used the obtained information to revise initial ideas about the kind of innovation needed. Then they took a revised innovation to clinicians and the lab team, and worked through several cycles of iterative prototyping, strengthening key features of the innovation, and dropping those less important.

XCo: then ... there's a synthesis period where you go away and you synthesize what you've seen, what you found, combined with what your inbound hypotheses were and come up with a set of observations or syntheses of these observations and, and opportunities. Uh, and you test that back with the clinicians... Two to three times and you're, in this case, you're prototyping the experience. Like what does a product looks like, what does it do, what are the features? The last step is you get to deploy it in the wild and start enrolling in this case, deploy and study it.

Throughout the process, end users - both patients and clinicians - had opportunity to contribute to the development of the platform, data collection approach, and clinical workspace, making the innovation more user friendly. The collaborators felt the innovation thereby would be more likely to be adopted and purchased, ultimately increasing viability of the business model.

\section{Piloting and Planning for Scale-up}

The collaborators prepared to pilot test the innovation in a clinical setting with 100 patients for effectiveness and cost-effectiveness. Data would reflect clinical outcomes and fidelity to the innovation for both patients and providers. Although a pilot increased time and cost, the partners believed it would encourage adoption and reduce potential adopters' fear of change

Simultaneously, XCo and the Innovation Lab began to work with their respective stakeholders to explore future scale up and innovation marketing. Within the hospital system, the Innovation Lab saw a need for buy-in from multiple stakeholders, including physicians, hospital leaders, and information technology. These stakeholders would need to perceive the innovation's connection to the hospital system's interests and larger strategy.
Moreover, the Innovation Lab recognized uptake of the innovation would be contingent on an available budget for the initial investment and their ability to demonstrate the innovation's applicability to multiple issues across clinical settings.

\section{Assessment of Process, Product, and Desired Outcomes}

Participants spoke about several characteristics of successful innovations for improving health systems, as well as the outcomes important for such innovations. They clearly articulated principles and features of partnerships between health systems and private innovation companies. These features, shown in Figure 3, are consistent with participants' narratives throughout the focus groups and detailed above.

\section{Consistent Trust and Transparency Throughout the Collaboration Development Process}

Given the risks and benefits of a collaboration, participants spoke of trust:

XCo: I think [trust] is actually essential to be able to move forward and do some exploration... without all the detailed terms. Like, there has to be some leap of faith. Otherwise... [activity] can get bogged down. Things lose momentum.

\section{Trust Was Particularly Essential in the Early Phases of the Collaboration}

Lab: At this point [when making the initial investments in XCo], the trust that I need to have in the [XCo $\mathrm{CEO}]$ and that he needed to have in me [Lab] was foundational. If it were purely transactional or one of us was really trying to screw the other, I think it would have fallen apart at this stage.

Building trust required transparency. Leaders of the partnering organizations were transparent about their own motivations and those of their respective stakeholders. The Lab was motivated to understand emerging capabilities in digital health and assess their relevance for the Cardiology Division, specifically the role of ambient sensing in remote patient monitoring programs in order to increase data completeness and quality. Other interests included the role of predictive analytics in managing the data flow from remote patient monitoring platforms and remote patient monitoring programs that provide substantive clinical value to patients. While these motivations did not necessarily conflict with the interests of XCo, neither were the Lab's interests necessarily in direct support of XCo's business objectives.

XCo's more business-focused motivations included securing actionable feedback about software products from intended clinical users, gaining a reference customer to boost confidence 
of other potential health systems and investors; and engaging in partnerships that lead to research and help establish brand reputation. All are essential for young "start ups."

Yet partners valued mutual benefit, thereby reducing perceived risks. They described this as a "leap of faith" partnership, thus engendering confidence: XCo: "I consider it a real success and a bit of a blueprint for how even other startups could be partnering early on." They also valued transparency between and within their organizations (up and down the hierarchy), regular communication, and the speed with which they both learned and resolved problems. The partners described this as an "ideal" partnership, absent factors that doom many collaborations.

\begin{abstract}
Lab: To learn fast, that means in my opinion, two things, a very tight cycle time and a lot of data you collect during that cycle time to then decide: change, continue, stop. And when you do that, you're acting on real-time information, you're acting on it quickly, you're learning from it.

You try and develop this minimally viable product and then you put it into the wild, get some input and then continue to make it better. That's done, you know, in a matter of sometimes days, but certainly shouldn't be more than a few weeks. That kind of timeline is unheard of in healthcare.
\end{abstract}

\section{Discussion and Implications}

This case study captures a process for developing implementable healthcare innovations through academic and corporate partnerships. Respondents gave varied perspectives - some complementary, some divergent; expressed a number of concerns; and were generally positive about the discussions - indicating their comfort expressing opinions. This study examined one partnership in one health system. While case studies have limited generalizability, they provide rich descriptions and yield working hypotheses for testing theory.

Several factors made this project appealing for our CTSA research. The project focused on two issues of high priority: (1) patients with complex and costly medical needs, and (2) digital innovations to capture patient-generated data $[1,3]$. The study afforded opportunity to examine partnerships working to innovate as the process evolved. Key players were actively engaged in a test bed phase, that is an iterative design phase or a process of receiving end user feedback to iterate the software design and functionality. The context was appropriate for examining the explanations and possible solutions from the perspectives of innovation and entrepreneurship (I\&E) and implementation science, and the processes they used.

Data revealed key questions that the partners worked to address, questions that could inform subsequent partnership endeavors and yield several implications for research translation in other settings and organizations. Key implementation science concepts that were "baked into" the conversations give carry important implications.

First, the partnership development was guided by clear, mutually valued goals - goals that align with clinical, health system, and implementation outcomes [16]. They sought to attain clinical outcomes of improving chronic heart failure symptoms. They shared a vision for the role of technology to accelerate the identification and response to clinical symptoms of chronic heart failure, thereby contributing to health system outcomes of timely, patient centered, and personalized care. A 2015 US Precision Medicine Initiative challenged healthcare organizations to capture data at the clinical encounter to inform ongoing system level improvements [17]. Partners wanted a product that the market would accept, adopt, and pay for-concepts consistent with both implementation science and entrepreneurship. They would explore the innovation's acceptability to clinicians and patients, capacity for sustainment and scale up, and costs - all implementation outcomes. As one XCo participant stated, “... if we can't get clinicians to adopt it, then it's all for nothing and there is no business."

Second, these data reflect the importance of deliberate formation and nurturing of partnerships. After a due-diligent, fact-finding process, the organizations worked to decide whether to move forward with the partnership. The innovation required collaboration among industry partners, technology developers, healthcare leaders, clinicians, patients, software developers, product designers, entrepreneurs/start-ups, private payers, and device makers [1]. The Innovation Lab wanted a co-creation opportunity with the private sector.

With partners acknowledging the possibility of failure, financial loss, and reputational damage, risk emerged as a third implication. Start-up companies have high failure rates, while medicine has a staunch commitment to "do no harm." Private sector entrepreneurs are likely more comfortable with risk than health systems. Start-up companies understand that, by definition, innovation requires risk. Partnering healthcare companies must accept the risk that the start-up company might fail; risk is a given, not career limiting for those involved with start-ups.

These partners regarded risk-harm reduction tension as a challenge to manage, not to be totally resolved. Complementing standard patient protection through IRB review, the Division and Lab managed risk through small "proofs of concept" initiatives to assess both efficacy and safety. Finally, the Lab constructed mitigation strategies such that any company's failure would not prevent accessing a beneficial digital health capability from another company.

Fourth, the co-creation observed here aligned with user/ human-centered design principles and "designing for dissemination" central to implementation science [18-21]; research impact [22] and entrepreneurship [1]. A "not fully developed" innovation was piloted with end users, whose feedback contributed to refinement. Developers wanted to shape only a "minimally viable product," to be tested for feasibility and acceptability, ensuring adopter influence on the final product. Iterative development, testing, and refining of innovations blurs boundaries between traditional phases of intervention development, testing, and implementation. End users - clinicians and providers - actively shaped the technology tools designed to improve patient care. Tools were designed with patient and clinician needs at the forefront, reflecting "design for dissemination," yielding the testable hypothesis that co-created products may be more readily adopted.

Consistent with both quality improvement [23] and implementation science [24-26], the work proceeded in phases - from initial exploration to scalability planning. Research translation is typically slow, whether via traditional research or tech transfer. The time from identifying a need to scaling a solution - known as "recognition to scale" or R2S - for digital innovations takes from 12 to 24 months [3]. While intervention development and scale can be achieved faster, the partners studied here felt that the iterative, co-creation processes was "not too slow, not too fast."

Because clinical and translational science is team-based, CTSA hubs have unique opportunity to leverage team science to design and support healthcare innovations beginning at project start [27]. This partnership between academia and commercial sectors for illustrates the need to expand traditional views of partnerships for implementing health system improvements [28]. Lessons from this project may inform other partnerships for innovation. 
Acknowledgements. Research reported in this publication was supported by the Washington University Institute of Clinical and Translational Sciences grant UL1TR002345 (VRM, BE, EKP) from the National Center for Advancing Translational Sciences (NCATS) of the National Institutes of Health (NIH). This research was also supported by funding from the National Institute of General Medical Sciences grant R25 GM116727-01A1 (ET), the National Institute of Mental Health grant R25 MH080916 (EKP, RL-H), the National Institute of Mental Health grant T32 MH019960 (RL$\mathrm{H})$, and the National Center for Advancing Translational Sciences grant UL1 TR000448 (ET).

Disclosures. Dr. Maddox discloses current grant funding from the NIH NCATS (1U24TR002306-01: A National Center for Digital Health Informatics Innovation) and honoraria and/or expense reimbursement in the past 3 years from Sentara Heart Hospital (Dec 2018), the Henry Ford health system (March 2019), and the University of California San Diego (Jan 2020). He is currently employed as a cardiologist and the executive director of the Healthcare Innovation Lab at BJC HealthCare/Washington University School of Medicine. In this capacity, he is advising Myia Labs, for which his employer is receiving equity compensation in the company. He is receiving no individual compensation from the company. He is also a compensated director for a New Mexico-based foundation, the J.F Maddox Foundation. Mr. MacGibbon owns equity in Myia Labs. The remaining authors have no conflicts of interest to declare.

\section{References}

1. Hostetter M, Klein S, McCarthy D, Hayes SL. Findings from a survey of health care delivery innovation centers. The Commonwealth Fund [Internet], 2015 [cited Oct 23, 2020]. (https://www.commonwealthfund. org/publications/publication/2015/apr/findings-survey-health-care-deliveryinnovation-centers)

2. O'Connor SJ. Enhancing the depth and breadth of healthcare services in communities: Insights, innovations, and applications. BMC Health Services Research 2017; 17: 1-38. doi: 10.1186/s12913-017-2338-5.

3. American Hospital Association. Executive Report, AHA and AVIA Digital Innovation Survey. 2017.

4. Shah SP, McCourt L, Jakobson K, Saddington A, Harvey K, Schulman KA. Leading change: a national survey of chief innovation officers in health systems. Health Management Policy and Innovation 2018; 3: 1-15.

5. Dolor RJ, Proctor EK, Stevens KR, Boone LR, Meissner P, Baldwin LM. Dissemination and implementation science activities across the Clinical Translation Science Award (CTSA) Consortium: Report from a survey of CTSA leaders. Journal of Clinical and Translation Science 2020; 4: 188-194. doi: 10.1017/cts.2019.422.

6. Leppin AL, Mahoney JE, Stevens KR, et al. Situating dissemination and implementation sciences within and across the translational research spectrum. Journal of Clinical and Translational Science 2020; 4: 1-7. doi: 10. 1017/cts.2019.392.

7. Powell BJ, Waltz TJ, Chinman MJ, et al. A refined compilation of implementation strategies: results from the Expert Recommendations for Implementing Change (ERIC) project. Implementation Science 2015; 10: 1-14. doi: 10.1186/s13012-015-0209-1.

8. Balasubramanian B, Cohen DJ, Davis MD, et al. Learning evaluation: blending quality improvement and implementation research methods to study healthcare innovations. Implementation Science 2015; 10: 1-11. doi: 10.1186/s13012-015-0219-z.

9. Thorp H, Goldstein B. Engines of Innovation: The Entrepreneurial University in the Twenty-First Century. Chapel Hill, NC: The University of North Carolina Press, 2010.

10. Divakaran D. Engaging with academic medical centers for digital innovation. Healthcare Information and Management Systems Society [Internet], 2020 [cited February 17, 2021]. (https://www.himss.org/ resources/engaging-academic-medical-centers-digital-innovation)
11. Mann DM, Chokshi SK, Lebwohl R, et al. Building digital innovation capacity at a large academic medical center. NPJ Digital Medicine 2019; 2. doi: 10.1038/s41746-019-0088-y.

12. Molloy SJ. Innovation Labs in Healthcare: A Review of Design Labs as a Model for Healthcare Innovation. Toronto, Canada: OCAD University, 2018.

13. Kumar J. Digital transformation of health care delivery in academic medical centers. Digital Information and Transformation: MBA Student Perspectives [Internet], 2018 [cited February 17, 2021]. (https://digital. hbs.edu/platform-digit/submission/digital-transformation-of-health-caredelivery-in-academic-medical-centers/)

14. Yin RK. Case Study Research and Applications: Design and Methods. Los Angeles, CA: SAGE Publications, 2017.

15. Crowe S, Cresswell K, Robertson A, et al. The case study approach. BMC Medical Research Methodology 2011; 11. doi: 10.1186/1471-228811-100.

16. Proctor EK, Silmere H, Raghavan R, et al. Outcomes for implementation research: conceptual distinctions, measurement challenges, and research agenda. Administration and Policy in Mental Health and Mental Health Services Research 2011; 35: 65-76. doi: 10.1007/s10488-010-0319-7.

17. Chambers DA, Feero WG, Khoury MJ. Convergence of implementation science, precision medicine, and the learning health care system: A new model for biomedical research. Journal of the American Medical Association 2016; 315: 1941-1942. doi: 10.1001/jama.2016.386.

18. Brownson RC, Jacobs JA, Tabak RG, Hoehner CM, Stamatakis KA. Designing for dissemination among public health researchers: findings from a national survey in the United States. American Journal of Public Health 2013; 103: 1693-1699. doi: 10.2105/AJPH.2012.301165.

19. Lyon AR, Bruns EJ. User-centered redesign of evidence-based psychosocial interventions to enhance implementation - Hospitable soil or better seeds? JAMA Psychiatry 2019; 76(1): 3-4. doi: 10.1001/jamapsychiatry. 2018.3060.

20. Dopp AR, Parisi KE, Munson SA, Lyon AR. A glossary of user-centered design strategis for implementation experts. Translational Behavioral Medicine 2019; 9(6): 1057-1064. doi: 10.1093./tbm/iby119.

21. Landis-Lewis Z, Kononowech J, Scott WJ, et al. Designing clinical practice feedback reports: three steps illustrated in Veterans Health Affairs longterm care facilities and programs. Implementation Science 2020; 15(7): 1-13. doi: 10.1186/s13012-019-0950-y.

22. Greenhalgh T, Jackson C, Shaw S, Janamian T. Achieving research impact through co-creation in community-based health services: literature review and case study. Millbank Quarterly 2016; 94(2): 392-429. doi: 10.1111/ 1468-0009.12197.

23. Cleghorn GD, Headrick LA. The PDSA cycle at the core of learning in health professions education. The Joint Commission Journal of on Quality and Patient Safety 1996; 22(3): 206-212. doi: 10.1016/s10703241(16)30223-1.

24. Aarons GA, Hurlburt M, Horwitz SM. Advancing a conceptual model of evidence-based practice implementation in public service sectors. Administration and Policy in Mental Health and Mental Health Services Research 2011; 38: 4-23. doi: 10.1007/s10488-010-0327-7.

25. Moullin JC, Dickson KS, Stadnick NA, Rabin B, Aarons GA. Systematic review of the Exploration, Preparation, Implementation, Sustainment (EPIS) framework. Implementation Science 2019; 14: 1-16. doi: 10.1186/ s13012-018-0842-6.

26. McCreight MS, Rabin BA, Glasgow RE, et al.. Using the Practical, Robust Implementation and Sustainability Model (PRISM) to qualitatively assess multilevel contextual factors to help plan, implement, evaluate, and disseminate health services programs. Translational Behavioral Medicine 2019; 9: 1002-1011. doi: 10.1093/tbm/ibz085.

27. Rolland B, Burnside ES, Voils CI, Shah MN, Brasier AR. Enhancing reproducibility using interprofessional team best practices. Journal of Clinical and Translational Science 2020. doi: 10.1017/cts.2020.512.

28. Chambers DA, Azrin ST. Research and services partnerships: partnership: a fundamental component of dissemination and implementation research. Psychiatric Services 2013; 64: 509-511. doi: 10.1176/appi.ps. 201300032. 